
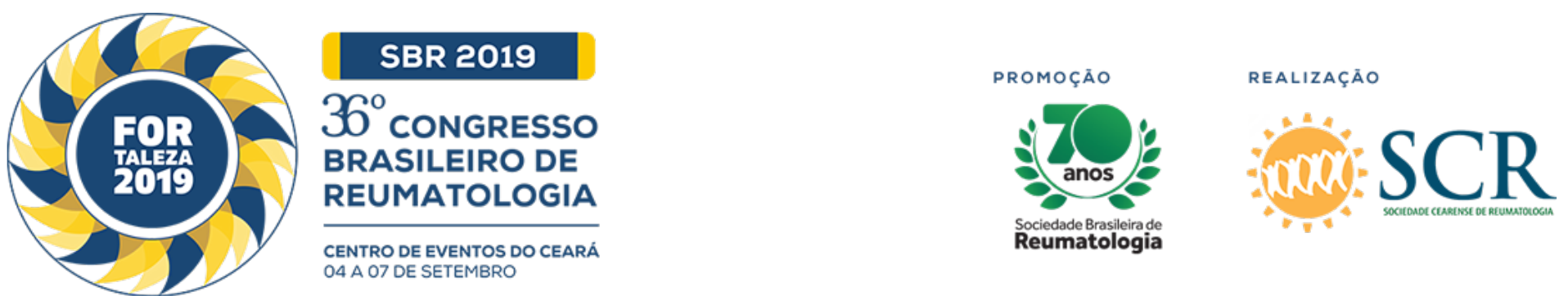

\title{
CLINICAL RELEVANCE OF SELF-REPORTED VISUAL ANALOGUE SCALE AND FUNCTIONAL CAPACITY IN PATIENTS WITH SYSTEMIC AUTOIMMUNE MYOPATHIES
}

Rafael Giovani Misse (Faculdade de Medicina FMUSP, Universidade de Sao Paulo, SP, Brasil (BR), São

Paulo, SP, Brasil), Alexandre Moura dos Santos (Faculdade de Medicina FMUSP, Universidade de Sao

Paulo, SP, Brasil (BR), São Paulo, SP, Brasil), Samuel Katsuyuski Katsuyuski Shinjo (Faculdade de Medicina FMUSP, Universidade de Sao Paulo, SP, Brasil (BR), São Paulo, SP, Brasil)

\section{BACKGROUND}

Muscle strength is one of the main measurements to evaluate the disease status and self-reported quality of life in patients with systemic autoimmune myopathies (SAMs). However, even among patients in whom the disease has stabilized and who have maintained muscle strength according to physician exams, quality of life has been impaired, mainly due to their muscles' health status. Therefore, the objective of the study was to analyze the correlation between muscle strength and functional and aerobic capacities in clinical and laboratory stable SAMs.

\section{MATERIALS AND METHODS}

A cross-sectional, single-center study was conducted between 2016 and 2018. A total of 13 consecutive adult patients with clinical and laboratory stable and defined SAMs were selected, 11 with dermatomyositis (EULAR/ACR classification criteria 2017) and 2 with antisynthetase syndrome (Connors et al., 2010). Limb strength was assessed by Manual Muscle Testing, MMT8)(0-80) physical examens and with the one-maximum repetition test in bench press (upper-limbs) and horizontal leg press (lower-limbs). Aerobic and functional capacities were assessed with the treadmill maximum test and the Healthy Assessment Questionnaire (HAQ)(0.00-3.00), respectively. Other applied exams were the physician and patients' Visual Analogue Scale (VAS)(0-10cm), Myositis Disease Activity Assessment VAS (MYOACT)(060), Timed-Stand Test (TST) and Timed Up-and-Go (TUG) test, as well as serum levels of creatine phosphokinase. Data were expressed as mean \pm standard deviation, median (interquartile 25th-75th) and Spearman correlation (rho).

\section{RESULTS}

The patients' mean age and disease duration were $45.8 \pm 1.8$ and $5.9 \pm 0.9$ years, respectively. All patients had stable disease: MMT-8 80 (80-80), HAQ 0.00 (0.00-0.30), patient's VAS 0.0 (0.0-0.5), creatine phosphokinase of $97.0(80.7-134.2) \mathrm{U} / \mathrm{L}$, MYOACT $0.0(0.0-0.3)$ Twelve patients were using at least one immunosuppressive drug, and one-third of them were using prednisone at the mean dose of 2.5 (0.0$5.0) \mathrm{mg} /$ day. The VO2 peak maximum was $18.6 \pm 1.1 \mathrm{~mL} / \mathrm{kg} / \mathrm{min}$, bench press of $22.2 \pm 2.0 \mathrm{~kg}$, leg press $51.0 \pm 4.8 \mathrm{~kg}$, TUG $7.1 \pm 0.2 \mathrm{~s}$, TST $13.0 \pm 0.6$ repetitions. The bench press correlated only inversely with patients' VAS (rho $=-0.69, \mathrm{P}=0.004)$ and $\mathrm{HAQ}(\mathrm{rho}=-0.59, \mathrm{P}=0.013)$. The leg press did not correlate with any parameters (HAQ, VAS, MYOACT, VO2 peak maximum, TST, TUG or creatine phosphokinase).

\section{CONCLUSION}

Even at low rates, the HAQ and patients' VAS values showed an inverse correlation with the parameters of muscle strength, not observed by medical evaluation (MMT8 and creatine phosphokinase) but by onemaximum repetition test of the upper-limbs. Therefore, it is relevant to valorize the patients' self-reported quality of life in clinical practice, even in those with clinical and laboratory stable disease. 